\title{
Involvement of very long fatty acid-containing lactosylceramide in lactosylceramide-mediated superoxide generation and migration in neutrophils
}

\author{
Kazuhisa Iwabuchi • Alessandro Prinetti • \\ Sandro Sonnino - Laura Mauri • Toshihide Kobayashi • \\ Kumiko Ishii • Naoko Kaga • Kimie Murayama • \\ Hidetake Kurihara • Hitoshi Nakayama • \\ Fumiko Yoshizaki • Kenji Takamori • Hideoki Ogawa • \\ Isao Nagaoka
}

Published online: 26 January 2008

(C) Springer Science + Business Media, LLC 2008

\section{Erratum to: Glycoconj J}

$$
\text { DOI 10.1007/s10719-007-9084-6 }
$$

The original version of this article unfortunately contained a mistake. The presentation of Fig. 3a was incorrect. The corrected figure is given below.

The online version of the original article can be found at http://dx.doi. org/10.1007/s10719-007-9084-6.

K. Iwabuchi $\cdot$ H. Nakayama $\cdot$ F. Yoshizaki $\cdot$ K. Takamori $\cdot$

H. Ogawa

Institute for Environmental and Gender-specific Medicine,

Juntendo University Graduate School of Medicine,

2-1-1 Tomioka, Urayasu-shi,

Chiba 279-0021, Japan

K. Iwabuchi $(\bowtie)$

Infectious Control Nursing,

Juntendo University Graduate School of Health Care and Nursing,

Chiba, Japan

e-mail: iwabuchi@med.juntendo.ac.jp

A. Prinetti $\cdot$ S. Sonnino $\cdot$ L. Mauri

Center of Excellence on Neurodegenerative Diseases,

Department of Medical Chemistry,

Biochemistry and Biotechnology, University of Milano, Milan, Italy
T. Kobayashi $\cdot$ K. Ishii

Sphingolipid Functions Laboratory, Frontier Research System, RIKEN,

Saitama, Japan

N. Kaga $\cdot$ K. Murayama

Division of Proteomics and Biomolecular Science,

BioMedical Research Center,

Juntendo University Graduate School of Medicine,

Tokyo, Japan

H. Kurihara

Department of Anatomy,

Juntendo University Graduate School of Medicine,

Tokyo, Japan

\section{Nagaoka}

Department of Host Defense and Biochemical Research, Juntendo University Graduate School of Medicine,

Tokyo, Japan 
a

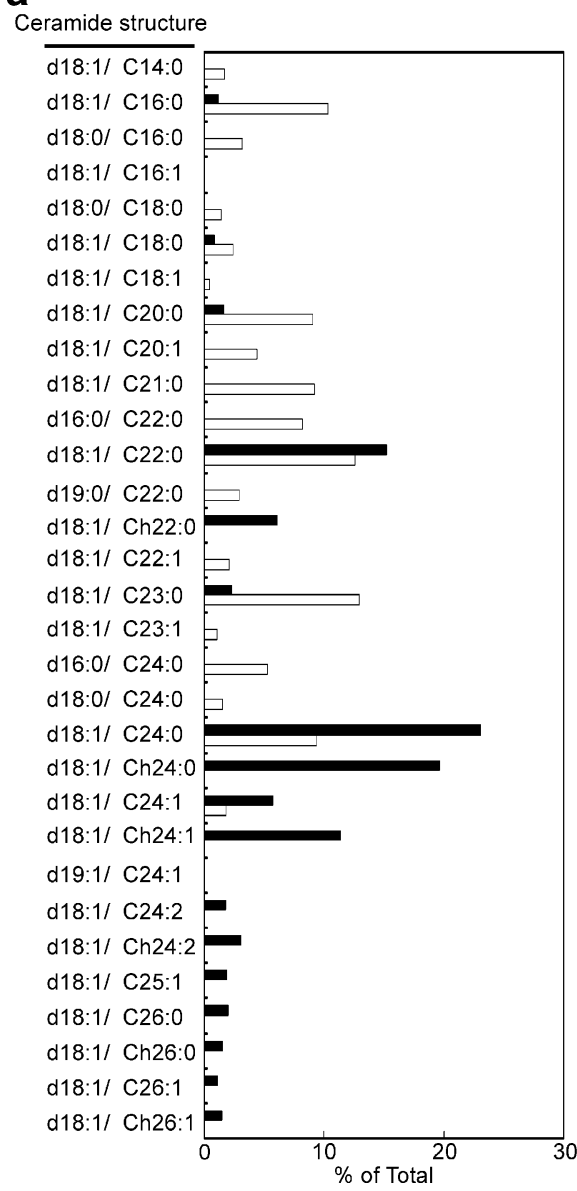

Fig. 3 Effects of natural LacCer loading on superoxide generation of D-HL-60 cells. (a) The molecular species of porcine blood cell- and bovine milk-derived LacCer. Porcine blood cell (closed bars) and bovine milk (open bars)-derived LacCer were subjected to LC-ESI$\mathrm{MS}^{\mathrm{n}}$ analysis as described in Materials and Methods. The amounts of b

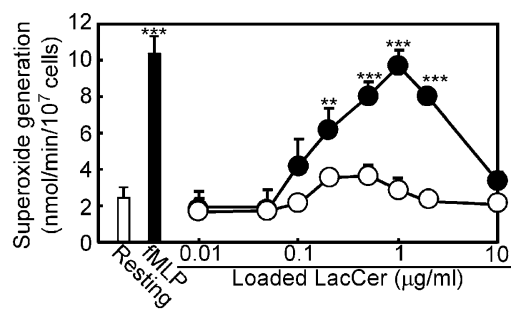

each LacCer molecule are shown as the percentages of total LacCer intensity. (b) D-HL-60 cells were incubated with porcine blood $(\bullet)$ or bovine milk (०)-derived LacCer at several concentrations at $20^{\circ} \mathrm{C}$ for 30 min. fMLP, $10^{-7} \mathrm{M}$ fMLP; Resting, $0.1 \%$ DMSO. Data show the means \pm SD of three independent experiments. $* * P<0.01, * * * P<0.001$ 